\title{
The Cepheid Distance to NGC 4414
}

\author{
Frank Thim \\ Astronomisches Institut der Universität Basel, Venusstr. 7, CH-4102 \\ Binningen, Switzerland
}

\begin{abstract}
A search for Cepheids in NGC 4414 has been performed using HST archive data. Accurate photometry for this galaxy is challenging due to its high surface brightness background with steep gradients and the rather low $\mathrm{S} / \mathrm{N}$ of stellar point sources. The photometry package ROMAFOT has been used, and the results compare favorably with published photometry reported by Turner et al. (1998). All eleven Cepheids were recovered and nine additional candidates were discovered. The resulting distance modulus of $31^{\mathrm{m}} \cdot 50 \pm 0^{\mathrm{m}} \cdot 27$ is only $0^{\mathrm{m}} 1$ larger than previously reported.
\end{abstract}

Images of NGC $4414\left(\alpha_{2000}=12^{\mathrm{h}} 26^{\mathrm{m}} 27^{\mathrm{s}} .5, \delta=+31^{\mathrm{o}} 13^{\prime} 29^{\prime \prime}\right)$ have been obtained with the HST Wide-Field and Planetary Camera 2 (WFPC2) camera. Twelve epochs in F555W and four epochs in F814W were obtained between 1995 April and June, with one additional F555W epoch in 1996 April. Each epoch in a given filter is composed of two sub-exposures of $1500 \mathrm{~s}$ and $1000 \mathrm{~s}$ duration, respectively. This allows the removal of cosmic ray hits.

The high surface brightness and strongly variable background make NGC 4414 one of the most difficult target fields of galaxies observed in the course of the HST Extragalactic Distance Scale Key Project (Kennicutt, Freedman, \& Mould 1995). To compare with the Key Project results ROMAFOT has been used in an iterative way to find as many variable stars as possible. The input catalogues for the PSF fitting of stars have been taken from deep frames. These are co-added images from different epochs, yielding a better $\mathrm{S} / \mathrm{N}$ ratio. These input catalogues have been used to perform photometry on every single epoch. The PSF has been established with relatively bright and isolated stars separately for every chip and filter. The resulting fitted images have been subtracted from the original frames. Then the PSF fitting has been performed on the residual images, and so on. In the end up to ten runs have been performed on successive residual frames. Artificial star tests show that the difference between input and measured magnitude follows the statistical errors in $V$ and $I$ with $50 \%$ completeness at $27^{\mathrm{m}}$ in $V$. New candidates have been found predominately near the inner region of the galaxy, where the photometry might be unreliable, or where crowding leads to problems of identification and matching between the two filters. These candidates are not of the same quality as the eight candidates used for the distance modulus determination.

The mean F555W and F814W magnitudes have then been converted to Johnson $V$ and Cousins $I$ using the equations given by Holtzman et al. (1995). For the $P-L$ relation only the eight bona fide candidates found both by Turner et al. (1998) and in this work have been considered. As illustrated in Fig. 1 the 

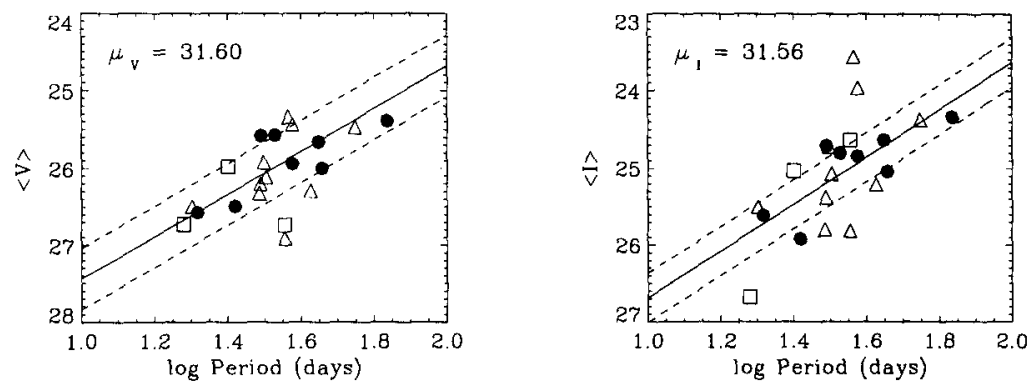

Figure 1. $\quad P-L$ relations for the Cepheids. The solid line represents the best fit with the canonical slope of -2.76 in $V$ and -3.06 in $I$. The envelope lines have been put at \pm 0.4 and $\pm 0.32 \mathrm{mag}$ from the solid line due to the intrinsic width of the instability strip. The eight best candidates have been plotted as filled circles. Candidates found by Turner et al. (1998), but with unreliable light curves as obtained with ROMAFOT, have been plotted as squares, new candidates as triangles.

adopted $P-L$ relations from Madore \& Freedman (1991) give apparent distance moduli of $\mu_{V}=31^{\mathrm{m}} 60 \pm 0^{\mathrm{m}} 11$ and $\mu_{I}=31^{\mathrm{m}} 56 \pm 0^{\mathrm{m}} 12$. Following Scheffler (1982), one has $A_{V}=0 .{ }^{\mathrm{m}} 10$ and $A_{I}=0 .{ }^{\mathrm{m}} 06$, and a true distance modulus of $31^{\mathrm{m}} 50 \pm 0.27$. Turner et al. (1998) derived a reddening-corrected distance modulus of $31^{\mathrm{m}} 41$ $\pm 0{ }^{\mathrm{m}} 17$ (random) $\pm 00^{\mathrm{m}} 16$ (systematic). As shown in Turner et al. (1998), the mean differences in magnitude of the Cepheid candidates for all WF chips between ALLFRAME and DoPHOT is $+0^{\mathrm{m}} 04 \pm 0.03$ in $V$ and $+0^{\mathrm{m}} 03 \pm 0.04$ in $I$. The mean difference of the Cepheid candidates between ALLFRAME and ROMAFOT is $-0.14 \pm 0.05$ in $V$ and $-0.14 \pm 0.06$ in $I$. Reasons for these differences might be the aperture correction or the use of those different photometry packages. Work to address this significant zero point offset is in progress.

Acknowledgments. I thank Lukas Labhardt for many fruitful discussions and suggestions, Gustav Tammann for the opportunity to take part in the conference, and the Swiss National Science Foundation for financial support.

\section{References}

Holtzman, J. A., Burrows, C. J., Casertano, S., et al. 1995, PASP, 107, 1065

Kennicutt, R. C., Freedman, W. L., \& Mould, J. R. 1995, AJ, 110, 1476

Madore, B. F. \& Freedman, W. L. 1991, PASP, 103, 933

Scheffler, H. 1982, in Landolt-Börnstein, Astronomy \& Astrophysics, Vol. 2c, ed. K. Schaifers \& H. H. Voigt (Berlin: Springer), 46

Turner A., Ferrarese, L., Saha, A., et al. 1998, ApJ, 505, 207 